\title{
La Independencia de la Auditoria
}

\author{
The Independence of the Audit
}

\author{
Sergio Demetrio Polo-Jiménez ${ }^{a}$, Graciela Muñoz-Arteaga ${ }^{b}$, Daniela Martín-Benítez $^{c}$
}

\begin{abstract}
:
The present work, seeks to know the level of influence that certain business characteristics maintain, with the independence index of the audit in the period 2010 to 2012, to reach the established objectives, a sample is analysed formed by 204 questionnaires of corporate best practices belonging to 68 companies listed on the Mexican Financial Market.
\end{abstract}

Keywords:

Audit, Independence of the Audit, Financial Market

\section{Resumen:}

El presente trabajo, pretende conocer el nivel de influencia que mantienen determinadas características empresariales, con en el índice de independencia de la auditoria en el periodo 2010 a 2012, Para alcanzar los objetivos establecidos, se analiza una muestra formada por 204 cuestionarios de mejores prácticas corporativas pertenecientes a 68 empresas cotizadas en el mercado financiero mexicano.

\section{Palabras Clave:}

Auditoria, Independencia de la Auditoria, Mercado Financiero

\section{Introducción}

El International Accounting Standards Board (IASB), es una organización privada sin fines de lucro, constituida en el año 2001, es la encargada de emitir y desarrollar la normatividad internacional en materia contable y financiera, cuyos objetivos se enlistan a continuación:

- Desarrollar, buscando el interés público, un único conjunto de normas contables de carácter global que sean de alta calidad, comprensibles y de cumplimiento obligado, que requieran información de alta calidad, transparente y comparable en los estados financieros y en otros tipos de información financiera, para ayudar a los participantes en los mercados de capitales de todo el mundo, a otros usuarios, a tomar decisiones económicas.

- Promover el uso y la aplicación rigurosa de tales normas.

- Cumplir con los objetivos, teniendo en cuenta, cuando se requiera, las necesidades especiales de entidades pequeñas y medianas y de economías emergentes, y

- Llevar a la convergencia entre las normas contables nacionales y las Normas Internacionales de Contabilidad y las Normas Internacionales de Información Financiera, hacia soluciones de alta calidad.

En este sentido, el IASB ha procurado el fortaleciendo de la independencia del auditor en su actuación profesional en los ámbitos tanto públicos como privados, a través de

\footnotetext{
a Autor de correspondencia, Universidad Autónoma del Estado de Hidalgo, Instituto de Ciencias Económico Administrativas de la UAEH, ORCID https://orcid.org/0000-0002-6137-5549, sjimenez@uaeh.edu.mx

b Universidad Autónoma del Estado de Hidalgo, Instituto de Ciencias Económico Administrativas de la UAEH, arteagagraciela03@yahoo.com.mx
} 
la emisión del Código de Ética para Profesionales de la Contabilidad emitido por el International Ethics Standards Board of Accountants (IESBA), específicamente en el apartado $A$, que versa sobre la aplicación general del código en sus puntos relativos a:

- Integridad

- Objetividad

- Competencia y diligencia profesional

- Confidencialidad

- Comportamiento profesional

El apartado B, menciona los aspectos personales y profesionales del contador público, tales como:

- Nombramiento profesional

- Conflictos de interés

- Segundas opiniones.

- Honorarios y otros tipos de remuneración

- Marketing de servicios profesionales

- Regalos e invitaciones

- Custodia de los activos de un cliente

- Objetividad-Todos los servicios

Independencia- encargados de auditoria y de revisión

- Independencia - Otros encargados que proporcionan un grado de seguridad

El Apartado C, especifica aspectos relativos a la relación empresa contador público, tales como:

- Conflictos de potenciales

- Preparación y presentación de información

- Actuación con suficiente especialización

- Intereses financieros

- Incentivos

Por su parte en México, el Instituto Mexicano de Contadores Públicos (IMCP) al igual que el IASB, emite las normas de actuación profesional para los contadores públicos contenidas en el Código de Ética Profesional (Documento que contiene la normatividad respecto a la actividad profesional del Contador integrado por 4 apartados y 24 aspectos específicos, por último, el relativo a las sanciones, este documento es de seguimiento obligatorio a partir del 1 de enero de 2012 para los auditores registrados).

En base a lo anterior el presente trabajo de investigación tiene como finalidad establecer si la independencia del auditor es una causa influyente en la determinación de la utilidad de las empresas mexicanas cotizadas en la Bolsa de Valores (años 2010-2012), con la finalidad de que dichas empresas obtengan mayor credibilidad ante sus inversores.

\section{Evidencia empírica}

Con la finalidad a conocer los estudios que tratan de encontrar la relación que existe entre los mecanismos del gobierno corporativo y el desarrollo de las organizaciones alrededor del mundo en especial, la función de auditoria, abordaremos aquellos estudios que de manera general investigan el nivel de influencia que mantiene el gobierno corporativo en el performance organizacional.

Sarkar, Sarkar y Kaustav (2012), construyen un índice que permite examinar la evolución de la situación de gobierno corporativo en la India, durante un período de tiempo en que ha existido un gran número de reformas a los conceptos que integran los mecanismos del gobierno corporativo en este país. Para cumplir con los objetivos planeados, se analiza una muestra formada por 500 empresas listadas en la bolsa de valores de la India, analizando para ello 4 dimensiones del código de prácticas societarias, entre ellas, la función de auditoría. Sus resultados muestran que existe una fuerte asociación estadística significativa entre el índice de gobierno corporativo y el desarrollo que tienen las empresas de la muestra, así mismo se resalta la importancia del gobierno corporativo en la valoración y crecimiento de confianza por parte de los inversores.

Así mismo, el trabajo realizado por Javaid y Saboor (2015), analizan una muestra formada por 58 empresas manufactureras cotizadas en el mercado financiero de Karachi en el periodo de 2009-2013, con el propósito de conocer empíricamente la relación que guarda el gobierno corporativo y el performance organizacional en términos financieros, así como el desempeño del mercado medido por retorno de los activos y recursos propios. A través de la aplicación de un modelo de regresión lineal, sus resultados les permiten afirmar la existencia de una relación significativa de tipo positivo entre el índice de gobierno corporativo (función de auditoría) y el performance de las empresas analizadas, lo que significa que, a mayor gobierno corporativo aplicado, mayor es el comportamiento financiero, el retorno de los activos y los recursos propios.

Otros estudios en el mismo año, también encuentran evidencia significativa, sobre la relación que mantiene la auditoria como mecanismo de gobierno corporativo y el desempeño organizacional, tal como lo presenta el estudio de Abulgasem et al. Quienes analizan una muestra formada por 25 empresas de Malasia

\section{Muestra}

Con la finalidad de alcanzar los objetivos del presente trabajo de investigación, se obtuvieron los datos, del Código de Mejores Prácticas Corporativas de las empresas que cotizan en la Bolsa Mexicana de Valores de los ejercicios de 2010, 2011 y 2012, correspondientes a 68 empresas de los sectores empresariales tales como 
Materiales, Industriales, de Servicios y Bienes de Consumo no Básico de Salud, de Productos de Consumo Frecuente y de Telecomunicaciones. El total de cuestionarios aplicados fue de 204, (Tabla 1).

\begin{tabular}{lcc}
\hline \multicolumn{1}{c}{ Sector } & $\begin{array}{c}\text { Número de } \\
\text { empresas }\end{array}$ & $\begin{array}{c}\text { Total de } \\
\text { cuestionarios } \\
\text { de mejores } \\
\text { prácticas } \\
\text { corporativas }\end{array}$ \\
\hline Industrial & 22 & 63 \\
Materiales & 11 & 33 \\
Productos & 14 & 42 \\
Consumo Frecuentes & & 9 \\
Salud & 3 & 39 \\
Servicios y consumo & 13 & 18 \\
no básico & & 204 \\
\hline Telecomunicaciones & 5 & \\
\hline Total & 68 & \\
\hline
\end{tabular}

Tabla 1. Descripción de la muestra

\section{Análisis descriptivo}

A continuación, se dan a conocer los resultados descriptivos del índice de independencia de la auditoria, en función del sector, tamaño de la empresa.

En la Figura 1, se observa que las empresas que cotizan en el mercado financiero mexicano, correspondientes al sector de telecomunicaciones, tienen un índice de cumplimiento de $92.59 \%$ en la independencia del auditor a diferencia de las empresas de Productos de consumo que tienen un índice de $88.89 \%$.

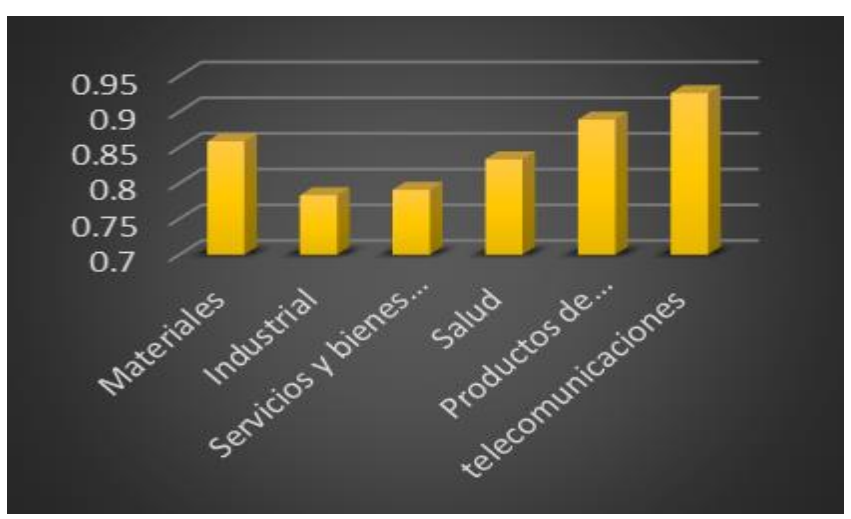

Figura 1. Independencia del auditor por sector

La Figura 2, presenta el índice de cumplimiento de la pregunta ¿Se abstiene de contratar aquellos despachos en los que los honorarios percibidos por la auditoria externa y otros servicios adicionales que presten a la sociedad, representen más del 10 por ciento de sus ingresos totales?, que muestra que el $92.65 \%$ de las empresas medianas que cotizan en el sistema financiero mexicano se abstienen de contratar aquellos despachos en los que los honorarios percibidos por la auditoria externa y otros servicios adicionales que presten a la sociedad, representen más del 10 por ciento de sus ingresos totales, a diferencia de las pequeñas y grandes que lo hacen en un $89.71 \%$.

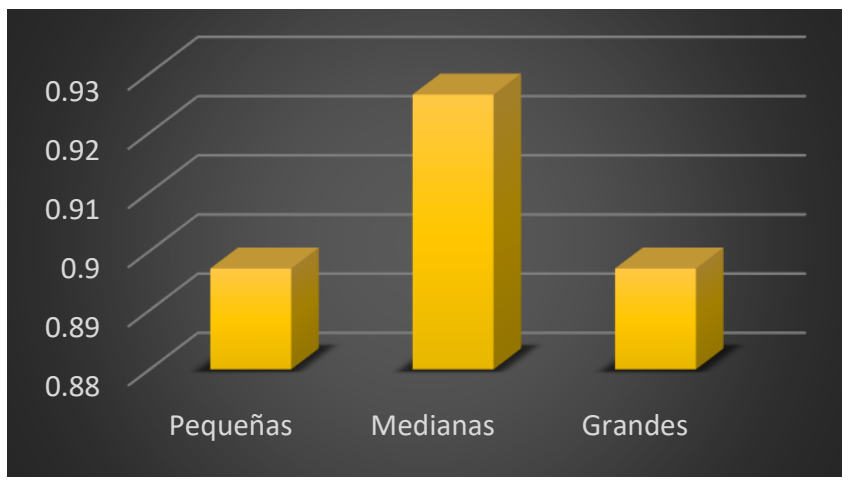

Figura 2. Contratación de servicios de auditoria externos

En la Figura 3, se observa que el $94.12 \%$ de las empres de tamaño pequeño y grande que cotizan en el sistema financiero mexicano, cambian al socio que dictamina los estados financieros así como a su grupo de trabajo, al menos cada 5 años, mientras que el $92.65 \%$ de las empresas que efectúa lo anterior son medianas.

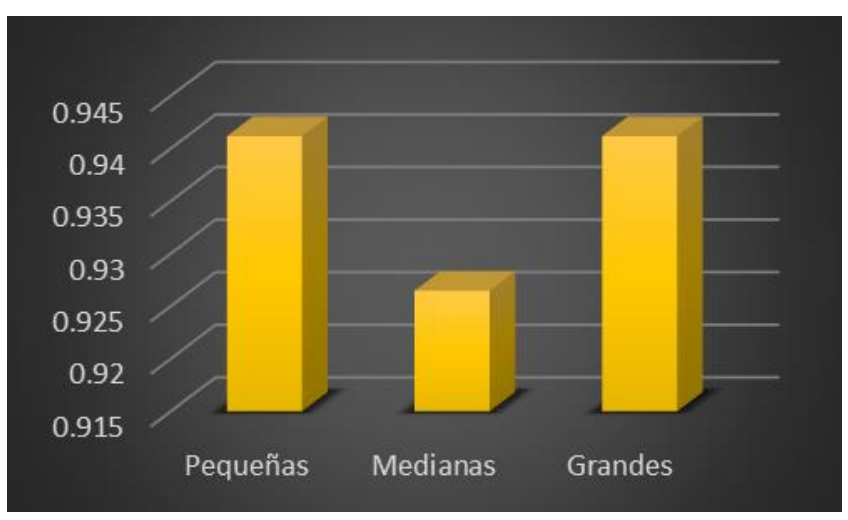

Figura 3. Índice de Cumplimiento de la pregunta ¿Se cambia al socio que dictamina los estados financieros así como a su grupo de trabajo, al menos cada 5 años?

La Figura 4, da evidencia respecto a que el $75 \%$ de las empresas grandes que cotizan en el sistema financiero mexicano, cuentan con una persona distinta a aquella que actúa como Comisario, en la firma del dictamen de auditoria a los estados financieros anuales de la sociedad, a diferencia de las empresas medianas, quienes solo lo hacen en un $61.76 \%$. 


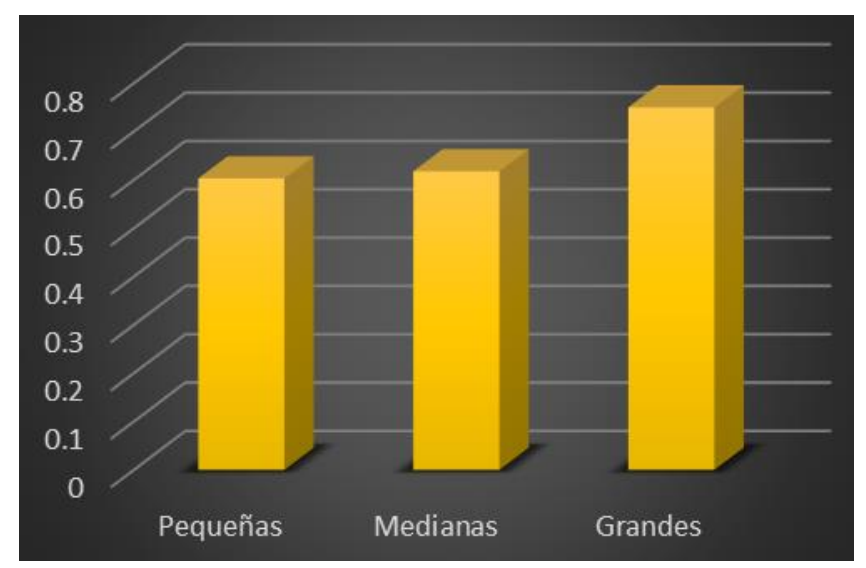

Figura 4. Índice de Cumplimiento de la pregunta ¿ La persona que firma el dictamen de la auditoria a los estados financieros anuales de la sociedad, es distinta de aquella que actúa como Comisario?

\section{Conclusiones}

El propósito del presente trabajo de investigación, ha recaído en la importancia de reconocer el nivel de influencia de determinados factores empresariales en en la independencia del auditor, al interior de una muestra de empresas que cotizan en el mercado financiero mexicano en el periodo 2010-2012. Los resultados, nos permiten suponer que de manera general la independencia del auditor en el periodo de estudio, se encuentra influenciada por el sector de pertenencia y el tamaño (medido por el total de sus activos al 31 de dicembre de cada año), lo que nos permite suponer que existe mayor atencion por parte de las autoridades regulatorias en sus facultades de comprobacion y fiscalizacion hacia determinados sectores empresariales, tambien pretendemos presumir que las empresas de mayor tamaño son aquellas que cuidadan de mejor manera la transparencia de sus operaciones pues uno de los requisitos para generar credibilidad en el mercado financiero es el informa de auditoria.

\section{Referencias}

[1] Sarkar, J.; Sarkar, S \& Sen, K. A Corporate Governance Index for Large Listed Companies in India (May 9, 2012). Available at SSRN: https://ssrn.com/abstract=2055091

http://dx.doi.org/10.2139/ssrn.2055091

[2] Javaid, F. Compliance with the treatment. Impact of Corporate Governance index on Firm Performance: evidence from Pakistani manufacturing 2015; 2(15): 1-21

[3] Mohamed Abulgasem, M.; Nurul Aini Muhamedb, N.; Mazna, N. The Influence of Corporate Governance, Financial Ratios, and Sukuk Structure on Sukuk Rating. Procedia Economics and Finance 2015; 31 $62-74$ 